\title{
The Electric Power Communication Transmission Network Risk Assessment Index System Research Based on AHP
}

\author{
Liu Chunyu ${ }^{1, a}$, Luo Jinyu ${ }^{2, b}$, Zhang Yongsheng ${ }^{3, c}$, Zhang Jie ${ }^{4, d}$ \\ ${ }^{1234}$ State Grid Inner Mongolia Eastern Power Co. Information Communication Ltd, China \\ Century Six Road, Hohhot city, Inner Mongolia province, China \\ a83211540@qq.com, bluojinyu@md.sgcc.com.cn, ccyberyosh@126.com, ${ }^{\mathrm{d}} 421403938 @ q q . c o m$
}

\begin{abstract}
Keyword: electric power communication transmission network (EPCTN);index system; analytic hierarchy process; weight
\end{abstract}

\begin{abstract}
By studying the SDH equipment of EPCTN, and the principles and methods of building the index system, combining with the actual situation of electric power communication operation, this paper establishes the index system of EPCTN risk assessment which includes target layer, rule layer, and index layer. Then we determine the distribution of the weight of each index according to the hierarchical grouping and the top-down approach, which provides the basis for the next step in risk analysis and calculation.
\end{abstract}

\section{Introduction}

Power Communication Network is the private network of power systems, including the support networks, business networks, switching network and transmission network, which is the physical part mainly composed by optical transmission equipment (SDH) and fiber composition [1]. EPCTN carries all business of the electricity production and management, the system is so large and the structure is so complex that any failure may have resulted in huge losses to the entire grid. Therefore, effectively assessing the risk profile of the power transmission network in time and point out the possible risk points, ensuring the business operate normally and improving the reliability of the entire network operation have a practical significance.

Due to the large quantity of network elements in the EPCTN and the equipment may come from different manufacturers, meanwhile the composition of the network topology structure is relatively complex, therefore, we need to make a comprehensive risk assessment on the transmission network [2]. First of all, we should start from the nets, namely the SDH optical transmission equipments, then analyzing their physical structure and the running performance data, so that we can determine the risk factors impacting the safe and stable operation of the equipments, and then construct the risk evaluation index system by using the analytic hierarchy process. At last, we may calculate the weights of each level's indicators by mathematical method which will be the foundation for the next step in risk analysis and calculation.

\section{Principles of determining index}

The establishment of the electric power communication transmission network risk assessment index system should satisfy some important principles what include the integrity, practicability, comparability, maneuverability, independence and so on [3].

a) The integrity is that the index system should fully cover all the factors which influence the risk of electric power communication transmission network, any of the factors should not be left out.

b) The practicality refers to the indexes which have been chosen should possess specific meaning, evaluation basis, and also the realistic foundation.

c) The comparability is to say that the rules should be consistent with the current ones as far as possible when designing the index names. The truth is that the stronger the comparability of indicators is, the higher the reliability of evaluation results is eventually. 
d) The operability expresses that the selected indicators are collected from the existing statistical data in principle, such as the network performance data, in addition, it is important to pay attention to the practicality and convenience when a few of the indicators need to be confirmed.

e) The independence requests to reduce the meaning overlap between the indicators as far as possible, for example, the indicators what are at the same level cannot contain each other, which can guarantee the indicators reflect the actual situation of equipment from different aspects.

\section{The establishment of hierarchical analysis model}

Analytic Hierarchy Process (AHP) is a kind of simple, flexible and practical multi-criteria decision-making method presented by the US operational research experts named Satty. T. $\mathrm{L}$ in the 70s [4]. It belongs to the category of subjective method, integrating people's subjective judgment, combining the qualitative analysis and quantitative calculation at the same time. It solves the difficult problem of analysis and calculation that only adopt quantitative method, so we employ the AHP in this article. The basic flow of AHP is shown in figure 1.

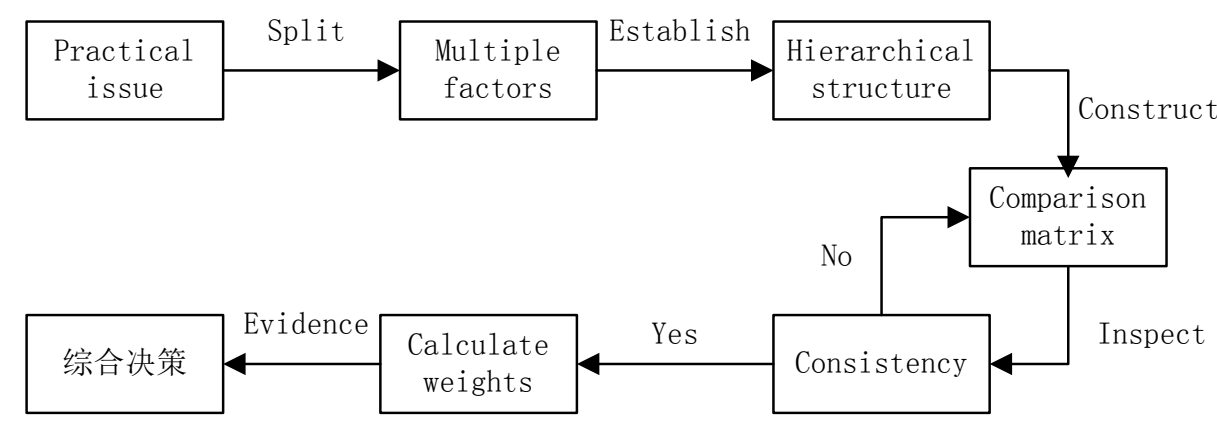

Fig.1 The basic flow of AHP.

\section{A. Construct hierarchical structure}

When conducting the risk assessment of EPCTN with the AHP, we should make the question methodical and hierarchical, and construct a hierarchical structure model first, as is shown in figure $2[5,6]$. The first layer is the target layer, it represents the risk value of SDH optical transmission equipments. Then the second is the rule layer, which is used to measure the risks by the confidentiality, integrity and availability of equipments. The third is indicator layer, including 8 first-level indexes, is defined as the risk points that influence the results of SDH equipments, and each point contains a number of risk factors, which form the second-level indexes, as in table 1 [7].

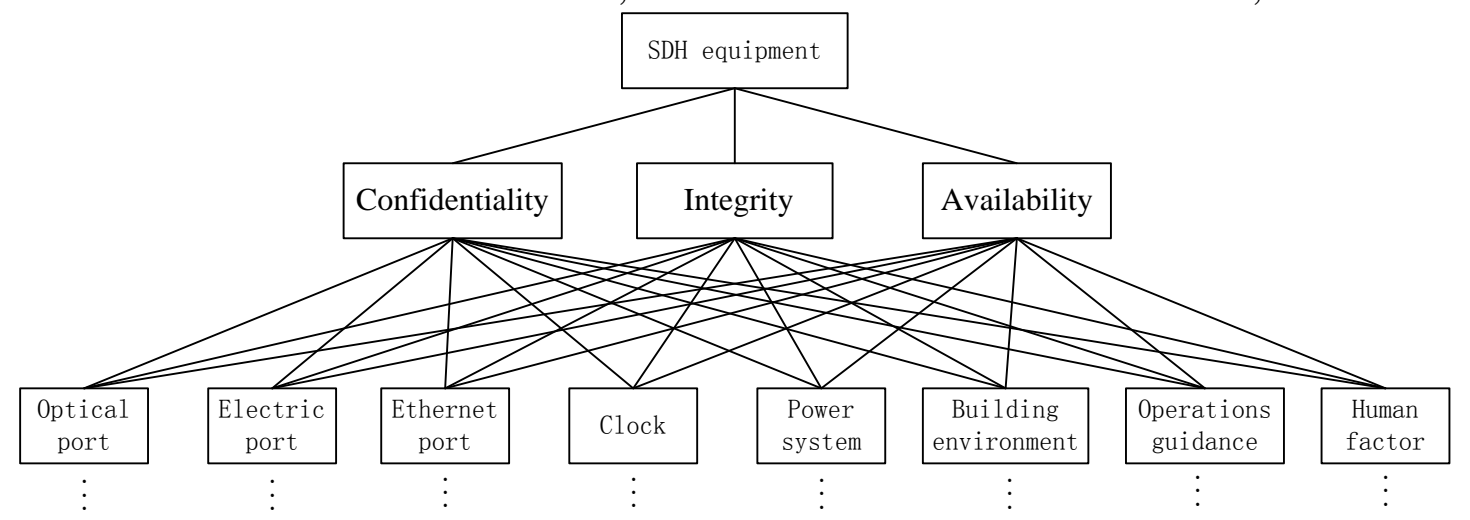

Fig. 2 The risk assessment hierarchy model. 
Table. 1 The mapping table of the first-level indexes and the second-level indexes

\begin{tabular}{|c|c|c|c|}
\hline First-level indexes & Second-level indexes & First-level indexes & Second-level indexes \\
\hline $\begin{array}{l}\text { Optical } \\
\text { port }\end{array}$ & $\begin{array}{c}\text { Transmit optical power } \\
\text { Receive optical power } \\
\text { AU pointer count } \\
\text { Bit error rate } \\
\text { Error second } \\
\text { Severely errored second } \\
\text { Optical channel frequency } \\
\text { Shake } \\
\text { Signal noise ratio }\end{array}$ & $\begin{array}{l}\text { Electric } \\
\text { port }\end{array}$ & $\begin{array}{c}\text { Current laser temperature } \\
\text { Current laser bias current } \\
\text { Laser back fire current } \\
\text { TU pointer count } \\
\text { PDH frequency deviation } \\
\text { PDH damping } \\
\text { PDH interface jitter } \\
\text { DSL error } \\
\text { Line error rate }\end{array}$ \\
\hline Clock & $\begin{array}{c}\text { Clock signal } \\
\text { Clock keeping precision } \\
\text { Clock frequency accuracy } \\
\text { Protection switch count } \\
\text { Protection switch duration }\end{array}$ & $\begin{array}{c}\text { Ethernet } \\
\text { port }\end{array}$ & $\begin{array}{l}\text { Handling capacity } \\
\text { Packet drop rate } \\
\text { Delay } \\
\text { _ } \\
\text { _ }\end{array}$ \\
\hline $\begin{array}{l}\text { Building } \\
\text { environment }\end{array}$ & $\begin{array}{c}\text { Temperature } \\
\text { Relative humidity } \\
\text { Air conditioning spare } \\
\text { Fire protection } \\
\text { Health situation }\end{array}$ & $\begin{array}{l}\text { Power } \\
\text { system }\end{array}$ & $\begin{array}{c}\text { Power voltage } \\
\text { Accumulator } \\
\text { Ground lead } \\
\text { Power supervision unit } \\
\text { Thunderbolt resistant }\end{array}$ \\
\hline $\begin{array}{l}\text { Operations } \\
\text { guidance }\end{array}$ & $\begin{array}{c}\text { Equipment maintenance } \\
\text { Spare parts } \\
\text { Defect elimination } \\
\text { Technical data } \\
\text { Emergency plan }\end{array}$ & $\begin{array}{l}\text { Human } \\
\text { factor }\end{array}$ & $\begin{array}{c}\text { Employee training } \\
\text { Personnel allocation } \\
\text { Age and education } \\
\text { Labor remuneration } \\
\text { Harmonious degree }\end{array}$ \\
\hline
\end{tabular}

\section{B. Weighting model construction}

It is important to give out the relative weight of elements that belong to the rule layer and index layer first when we start to analysis the risk of equipments, and calculate the relative weights with the traditional method AHP [8,9]. The specific approach is regarding some element of the upper layer as the judgment criteria, then compare the important degree of the lower elements one by one. At last, we need to confirm the relative important degree given out by the experts who are experienced and good sense, and then establish the judgment matrix of the elements, as in Eq 1:

$$
A=\left[\begin{array}{cccc}
a_{11} & a_{12} & \ldots & a_{1 n} \\
a_{21} & a_{22} & \ldots & a_{2 n} \\
\vdots & \vdots & & \vdots \\
a_{n 1} & a_{n 2} & \ldots & a_{n n}
\end{array}\right] .
$$

We adopt the 9 points a ratio scale in this paper based on the theoretical study proposed by the relevant scholars when comparing the relative important degree. It is said that the limit capacity of people distinguish the level of information is 7 plus or subtract 2 [10]. Namely the value of each elements in the judgment matrix is set between 1-9 or the reciprocal.

The experts can establish the judgment matrixes of rule layer and index layer based on the method referred above, we will take rule layer for example such as shown in table 2. 
Table 2. The judgment matrix of rule layer

\begin{tabular}{c|c|c|c}
\hline A & Confidentiality & Integrity & Availability \\
\hline Confidentiality & 1 & 2 & 3 \\
\hline Integrity & $1 / 2$ & 1 & 2 \\
\hline Availability & $1 / 3$ & $1 / 2$ & 1 \\
\hline
\end{tabular}

\section{The inspection of consistency and the calculation of the weights}

According to the principle of positive matrix, if the matrix A satisfy the Eq 2, and the matrix has the only nonzero largest eigenvalue, then we call the matrix A meeting completely consistent. But when experts make comparison between the two of the factors, it is unlikely to be completely consistent, which lead to the eigenvalue and eigenvector have a deviation. For example, it may be that a is more important than $\mathrm{b}, \mathrm{b}$ is more important than c and c is more important than $a$ which violates the logic. Such a judgment matrix going against the most basic consistency reflects the decision makers' logical confusion on measuring the importance of various factors and has no reference value for the final decision. So it is necessary to inspect the consistency of matrix A. If the judgment matrix doesn't satisfy the requirement of the consistency, it is necessary to modify it.

$$
b_{i j}=b_{i w} / b_{j w} .
$$

1) Making inspection of the judgment matrix, it needs to work out the largest eigenvalue $\lambda_{\max }$ and eigenvector $\mathrm{W}$. We often use the simple approximate method, because it is very difficult to take the eigenvalue and eigenvector through mathematical definition, especially when the order of the matrix is so high. For example, in this article the number of indexes in both the optical interface and the electricity port is 9 , so that is a 9 order matrix. In addition, the judgment matrix is the result of the qualitative comparison, so it is not necessary to make accurately calculation. The commonly used method is square root method and summation method, which is used to calculate the largest eigenvalue and the corresponding eigenvector of the matrix.

Using each line's factors of the matrix A multiplied by a column, then take the $\mathrm{n}$ roots of the result, $\mathrm{n}$ is the orders of judgment matrix. We can get the eigenvector $W_{i}$ and the largest eigenvalue $\lambda_{\max }$. As in Eq. 3 and Eq. 4.

$$
\begin{aligned}
& W_{\mathrm{i}}=\sqrt[n]{\prod_{j=1}^{n} a_{i j}}=\sqrt[3]{\prod_{j=1}^{3} a_{i j}} . \\
& \lambda_{\max }=\frac{1}{n} \sum_{i=1}^{\mathrm{n}} \frac{(A W)_{i}}{W_{i}}=\frac{1}{3} \sum_{\mathrm{i}=1}^{3} \frac{(A W)_{i}}{W_{i}} .
\end{aligned}
$$

So $W_{i}=(1.8171,1,0.5503)^{\mathrm{T}}$ and $\lambda_{\max }=3.0093$.

2) Calculating the consistency indicators $I_{C}$, as in Eq. 5.

$$
I_{C}=\left(\lambda_{\max }-n\right) /(n-1)=\left(\lambda_{\max }-3\right) / 2 .
$$

So $I_{C}=0.0047$. The greater the value of $I_{C}$, the worse consistency of the A matrix.

3) As the high orders of the judgment matrix, it is difficult to meet the consistency requirements. To calculate the consistency of different judgment matrix, we give out a 10 orders judgment matrix' average consistency indicators. As is shown in table 3: 
Table 3. The 10-order average consistency index table

\begin{tabular}{c|c|c|c|c|c|c|c|c|c|c}
\hline Order & 1 & 2 & 3 & 4 & 5 & 6 & 7 & 8 & 9 & 10 \\
\hline$I_{R}$ & 0 & 0 & 0.52 & 0.90 & 1.12 & 1.26 & 1.36 & 1.41 & 1.46 & 1.49 \\
\hline
\end{tabular}

In this article the order of the judgment matrix is 3 , so the average consistency indicator $I_{R}=0.52$. 4) Calculate the consistency ratio $R_{C}$, as in Eq 6 .

$$
R_{C}=I_{C} / I_{R} \text {. }
$$

So $R_{C}=0.0047 / 0.52=0.0090$. If the consistency ratio is 0 , the matrix $\mathrm{A}$ has completely consistent, while we don't need completely consistent in the real operation, when $R_{C}<0.1$ in theory, matrix A has a good consistent, otherwise it should be modified. It can be seen, the structured rule layer judgment matrix satisfy the consistency requirement.

To calculate the vector $W=\left[W_{1}, W_{2} \ldots W_{n}\right]$ and make it normalized, as in Eq 7.

$$
W_{\mathrm{i}}=W_{i} / \sum_{i=1}^{n} W_{i}
$$

So $W=(0.5396,0.2970,0.1634)^{\mathrm{T}}$. For the judgment matrix A accord with the consistent test, so the eigenvector of the judgment matrix is the one of the rule layer's weight vector, (confidentiality, integrity, and availability $)=\left(\begin{array}{lll}0.5396,0.2970,0.1634\end{array}\right)$.

\section{The calculation of composite weights}

When calculating the weight of the index layer' first level indicators, because of the influence to the three factors of the rule layer, we make the calculation easy. Considering that the influenced degree is divided into very low, low, medium, high, very high five grades, Its orders of magnitude are expressed by $0.1,0.3,0.5,0.7,0.9$, as in table 4 :

\begin{tabular}{c|c|c|c|c|c}
\hline $\begin{array}{c}\text { First level indexes } \\
\text { / Rule }\end{array}$ & Confidentiality & Integrity & Availability & $\begin{array}{c}\text { Effect to } \\
\text { the target }\end{array}$ & Normalization \\
\hline Optical port & 0.9 & 0.7 & 0.7 & 0.8079 & 0.1880 \\
\hline Electric port & 0.9 & 0.7 & 0.7 & 0.8079 & 0.1880 \\
\hline Ethernet port & 0.7 & 0.7 & 0.5 & 0.6673 & 0.1553 \\
\hline Clock & 0.7 & 0.5 & 0.5 & 0.6079 & 0.1415 \\
\hline Power system & 0.5 & 0.5 & 0.7 & 0.5327 & 0.1240 \\
\hline Building environment & 0.3 & 0.3 & 0.5 & 0.3327 & 0.0774 \\
\hline Operations guidance & 0.3 & 0.3 & 0.3 & 0.3000 & 0.0698 \\
\hline Human factor & 0.3 & 0.1 & 0.3 & 0.2406 & 0.0560 \\
\hline
\end{tabular}

We can get the composite weights of all the elements in first level layer, for the reason that the factors of secondary index layer are too many, and the method of weight calculation is the same to the rule layer, so we will not expatiate. When getting the secondary index's weight and multiplying it with the first index's weight value, we may obtain the target layer's composite weights from the secondary index. 


\section{Conclusion}

By building risk assessment hierarchy model of the optical transmission equipment in EPCTN, we calculated the index weights in the first and secondary indicator layers. Combined with the score of the performance parameters of SDH equipments, using the risk assessment algorithms: risk value = performance rating * risk factors' weight, we can make the dynamic risk assessment of the optical transmission equipment in the transmission network. Once the SDH equipments are in high risk level, we can discover the corresponding risk points in time, so as to take relative risk control measures and ensure the safety and reliable operation of the EPCTN.

\section{References}

[1] Gao Huisheng, Wang Xurui, Wang Huifang: A Comprehensive Security Evaluation Model of SDH Equipments in Power Communication Network Based on Bayesian Network. Modern Electric Power. Vol. 31-3 (2014). p. 68-73.

[2] Wang Feng. A Design and Research on Electric Power Communication Network Reliability Evaluation Index and Evaluation System. Communication Technology. (2014).

[3] Yuan Zhao, Jian Jiao, Zhao Tingdi: A Synthetic Risk Assessment Model Based on AHP. Reliability and Maintainability Symposium(RAMS). (2014). p. 1-6.

[4] Satty,T.L: The Analytc Hierarchy Process.New York: McGraw-Hill (1980).

[5] Zhu Xiping, Zhang Laibin, Liang Wei: Study on Index System of Risk Assessment for Centrifugal Compressor on AHP. Instrumentation and Measurement, Sensor Network and Automation (IMSNA). (2013). P. 138-140.

[6] Qi Feng, Li Qi, Han Qian, Du Yi, Qiu Xuesong. A Neural Network Based Method to Assess Electric Power Communication Network Risk.Journal of Beijing University of Posts and Telecommunications. Vol. 37-1 (2014). P. 90-93.

[7] Xu Jian, Guo Shaoyong. An Power Communication Network Mechanism Based on AHP. Telecommunications Technology. (2013). P. 73-75.

[8] Lu Xudong, Huang Fuliang, Song Wei. Electric Power Communication Network Risk Assessment Model. Telecommunications for Electric Power System. Vol. 31-325 (2010). p. 19-23.

[9] Su Yuke. Eletric Power Communication Network Reliability Evaluation Index System. Information and Communications. Vol. 11 (2014). P. 197-199.

[10] Gao Xuesheng, Chen Bing, Han Chunlei, Tang Yunshan. Research and Application on Power Communication Operations Management Risk Assessment. Electric Power ICT. Vol. 11-9 (2013). P. 49-53. 